\title{
Flight-related aneurysmal subarachnoid hemorrhage and its complications during and after landing
}

\begin{abstract}
It has been suggested that the risk of rupture of intracranial aneurysms may be fostered by climatic drops in barometric pressure, as also occurs to passengers subjected to the air pressure settings inside aircraft cabins. We present two cases of flight-related aneurysmal SAH followed by intracerebral rebleeding in the cabin (case 1) and carotid dissection after landing (case 2). Management and complications of in-flight subarachnoid hemorrhage, influence of decreased cabin pressure on the aneurysm rupture, timing of hemorrhage related to flight exposure and counseling of patients with unruptured aneurysms are discussed.
\end{abstract}

Keywords: SAH, Aneurysm, Flight, Cabins, Headache, Air travel, Atmospheric pressure

\author{
Volume 3 Issue I - 2015
}

E Pozzati, F Nicolini, A Fioravanti

IRCCS Institute of the Neurological Sciences of Bologna, Division of Neurosurgery, Bologna, Italy

Correspondence: A Fioravanti, IRCCS Institute of the Neurological Sciences of Bologna, Division of Neurosurgery, Bologna, Italy, Tel 05I-6225500,

Email Antonio.fioravanti@ausl.bo.it

Received: April 24, 20I5 | Published: October 08, 2015

\section{Introduction}

Approximately $5 \%$ of the adult population have cerebral aneurysms, but the average annual incidence of subarachnoid hemorrhage (SAH) occurs in a net minority of cases (about 10 in 100.000 people); ${ }^{1}$ besides hemorrhage, an increasing problem is represented by the management and counseling of patients with intact aneurysms.

Aneurysm rupture is multifactorial and depends on a number of congenital, pathological and environmental factors; in particular, it has been associated to climatic variations and drops in barometric pressure. ${ }^{1-6}$ The association between changes in atmospheric pressure and ruptured aneurysms could also be applied to air travelers subjected to a number of physical factors, including lowering of cabin pressure, that may adversely affect the cerebrovascular adaptations and the transmural pressure across the aneurysm. When compared to the impressive commercial air traffic, reports of in-flight SAH remain quite low, but the safety margins of air travel in patients with aneurysms are poorly understood. Two cases are presented of in-flight SAH aiming to a better understanding of complications related to air travel and to counseling of patients with intracranial aneurysm.

\section{Materials and methods}

\section{Case I}

This 48- years old left handed hypertensive woman had sudden headache and vomiting two hours after the take-off of a transatlantic flight from Italy to Argentina. In the following hours left hemiparesis and frank comatose state occurred. Due to the acute clinical worsening, a flight diversion was scheduled and an emergency landing was performed in Brazil. On hospital admission, the patient was in deep coma with left hemiplegia and GCS of 8/15. A CT-scan diagnosis of SAH complicated by right intracerebral temporal haematoma was made (Figure 1). Cerebral angiography demonstrated three aneurysms of the right middle cerebral artery, posterior communicating artery and anterior communicating artery. The past medical history revealed a previous SAH occurred 25 years before secondary to an aneurysm of the anterior communicating artery which had been wrapped in Italy when she was 25 year old.

At operation in Brazil, the right fronto-temporal flap was reopened, the hematoma evacuated and the three aneurysms successfully clipped. Post-operatively the patient improved but after three days she rapidly declined due to right temporal ischemia and edema. Her conditions gradually cleared after medical treatment and the return to Italy was planned 16 days after $\mathrm{SAH}$; control angiography was not performed. When admitted at our Hospital, her conditions worsened again; CT scan demonstrated a marked hydrocephalus and a wide fronto-temporal ischemia. A ventriculo-peritoneal shunt was inserted and her neurological conditions improved in the following days: at six months, she remained deeply hemiparetic and dysarthric.

\section{Case 2}

This 40-year old normotensive woman had sudden headache before landing of an intercontinental flight from Egypt to Italy which worsened in the shuttle of the airport.

On admission at our Hospital, CT scan demonstrated a focal SAH in the right pericarotid and sylvian cisterns and cerebral angiography showed a small "blister" aneurysm of the infero-medial portion of the right supraclinoid ICA (Figure 2). A right pterional craniotomy with anterior clinoidectomy was performed. A non saccular carotid aneurysm was found: clipping was not possible and wrapping of the arterial dilatation was performed. The exploration of the ICA and its branches did not reveal any changes. Postoperative course was uneventful. At postoperative control angiogram at two weeks the aneurysm was unchanged and stenosis of the right anterior cerebral and posterior communicating arteries due to vasospasm or dissection was present (Figure 3). At one year, the patients remained well and the narrowing was unchanged, confirming the hypothesis of a residual dissection.

\section{Discussion}

\section{Pathophysiology}

It has been suggested that the risk of rupture of intracranial aneurysms may be fostered by changes in barometric pressure. ${ }^{1-5}$ Admission clusters of patients with $\mathrm{SAH}$ following a drop in barometric pressure have been reported; ${ }^{1,3-5}$ changing climatic conditions preceding aneurysm rupture were characterized by a fall in maximum daily barometric pressure of greater than $55 \mathrm{mmHg} .{ }^{1}$ Atmospheric pressure is $760 \mathrm{mmHg}$ at sea level; commercial air craft cabins at a cruising altitude of $11000 \mathrm{~m}$ are pressurized at the atmospheric pressure found at 5000-8000ft (2000-2500 meters), equivalent to $632-552 \mathrm{mmHg} .{ }^{7,8}$ This hypobaric condition in air travel 
generally induces some hypoxemia, brain swelling and increase of cerebral blood flow. ${ }^{9}$ High altitude sickness represents the closest analogy to the neurological effects associated with air travel, but rapid airplane ascent and descent can trigger further adverse cerebrovascular changes.

Reports of in-flight SAH after rupture of saccular and dissecative aneurysms are very limited. Although the cause of aneurysmal rupture is multifactorial and in-flight SAH may represent a chance occurrence in this special stressing milieu, the decreased atmospheric pressure in the airplane cabin may have a causal relationship owing to changeable transmural pressure across the aneurysm wall, ${ }^{8-10}$ as demonstrated by the example of an empty bottle, closed during flight and then crushed by the pressure at ground level after descent.

The same mechanism has been advocated in patients with hemorrhage within brain tumors in association with long air travel. ${ }^{8,9}$ Under this aspect, it is interesting to note that hemorrhage tends to occur during descent or immediately after, which probably represents the most sensitive phase of flight. ${ }^{7-10}$

The effect of reactive increased cerebral blood flow related to decreased in blood oxygen saturation may contribute to in-flight aneurysm rupture, ${ }^{7}$ and to adverse consequences in terms of rebleeding and intracerebral hemorrhagic extension. Cerebral hypoxia may be associated also with significant increases in cerebral artery diameters, ${ }^{11}$ which may modify aneurysm haemodynamics and promote bleeding.

Some delay in the clinical effects of exposure to atmospheric pressure changes should be also considered: in season changes, aneurysmal rupture was significantly associated with marked variations in barometric pressure occurring from 24 to 72 hours before hemorrhage. ${ }^{1-6}$ Although the rapidity of in-flight atmospheric pressure changes may influence the aneurysm rupture particularly in the cabin, the risk should be extended to a period following arrival, also considering the mutual relationships among cruise altitude, travel frequency and flight duration. For instance, the repetitive and rapid pressurization/depressurization cycles that occur in "frequent flyers"and multi-stop flights may adversely affect the aneurysm wall.
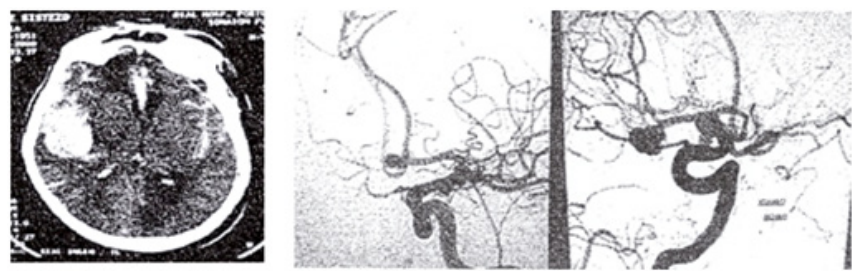

Figure I Case I: on the left CT-scan on hospital admission in Brazil shows a right temporal hematoma with diffuse SAH (left); preoperative angiogram in frontal (central) and lateral (right) projection shows multiple aneurysms (arrow) in the middle cerebral and anterior communicating arteries.
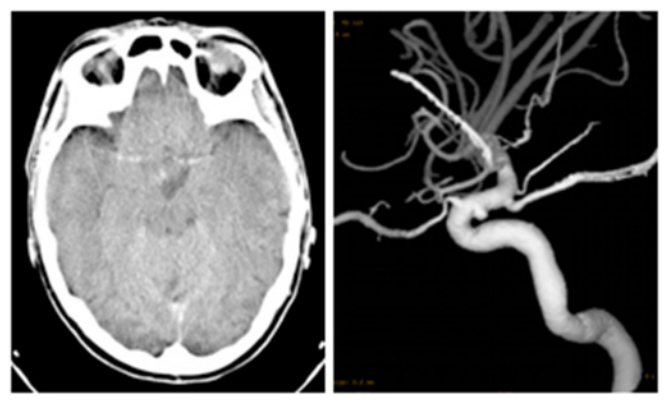

Figure 2 Case 2: CT-scan shows a SAH in sylvian fissure and right pericarotid cistern (left); carotid angiogram reveals a small "blister" aneurysm of the infero-medial portion of the right supraclinoid ICA (right).
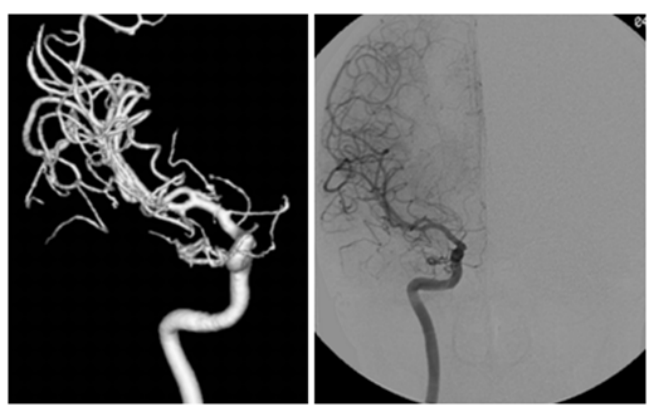

Figure 3 3D- Angiographic reconstruction shows stenosis of the right posterior communicating and anterior cerebral arteries (arrow) at postoperative angiogram two weeks after the operation related to possible dissection.

\section{Counseling}

The detection of unruptured cerebral aneurysms has recently increased due to the advances in non-invasive diagnostic techniques but the lifestyle of the affected people remains largely undetermined . Smoking, multiple aneurysms and hypertension are well established risk factors for rupture. Considering that most patients with intracranial aneurysms fly without complications, the risk of air travel is not sufficiently addressed in the believe that the risk in this special "milieu" conforms with that at ground level : reasonable limitations should be better identified adapting the indications of multicenter studies on unruptured intracranial aneurysms regarding size, shape and location to the risks of the flight environment. Even more restrictive safety margins should be applied to aviation personnel regarding in particular aneurysmal size and shape which may be adversely affected by the effects of changing transmural pressure. ${ }^{12}$

Our second patient may suggest that a special consideration of inflight sentinel headache and SAH occurring after landing is necessary. This case documents an unusual dissection of the supraclinoid ICA evolving from an initial blister aneurysm with focal leak to a late narrowing of the anterior cerebral artery (Figure 2 and 3). Blister aneurysms constitute a subgroup of carotid aneurysms with fragile wall which may be exposed to the risks of changing transmural pressure.

Unsafe flight indications represented by previous SAH and aneurismal multiplicity are clearly confirmed by the clinical course of our patient 1 . The clinical events during flight in this patient also suggest the occurrence of early rebleeding as demonstrated by the rapid clinical decline due to the intracerebral hematoma complicating the initial SAH.

Management of in-flight SAH should be primarily focused on the prevention of rebleeding and aircraft diversion is mandatory in view of the difficult medical care and adverse cerebrovascular adaptations related to $\mathrm{SAH}$ in this special environment.

A retrospective study with internationally acquired series of SAH may help a better understanding of flight complications both in its different steps (take off, cruise, landing) and in the short term postflight consequences at ground level, ${ }^{13,14}$ which may be not enough considered in their relationships with air-travel.

\section{Ackowledgments}

None. 


\section{Conflicts of interst}

None.

\section{References}

1. Chyatte D, Chen TL, Bronstein K, et al. Seasonal fluctuation in the incidence of intracranial aneurysm rupture and its relationship to changing climatic conditions. J Neurosurg. 1994;81(4):525-530.

2. Buxton N, Liu C, Dasic D, et al. Relationship of aneurysmal subarachnoid hemorrhage to changes in atmospheric pressure: results of a prospective study. J Neurosurg. 1994;95(3):391-392.

3. Jehle D, Moscati R, Frye J, Reich N The incidence of spontaneous subarachnoid hemorrhage with change in barometric pressure. Am J Emerg Med. 1994;12(1):90-91.

4. Lejeune J, Vinchon M, Amouyel P, et al. Association of occurrence of aneurysmal bleeding with metereological variations in Northern France. Stroke. 1994;25(2):338-341.

5. Forstad H Subarachnoid hemorrhage and atmospheric pressure. $J$ Neurosurg. 2002;96(5):974-975.

6. Landers AT, Narotam PK, Govender ST, et al.The effect of changes in barometric pressure on the risk of rupture of intracranial aneurysm. $\mathrm{Br} \mathrm{J}$ Neurosurg. 1997;11(3):191-195.
7. Cui V, Kouliev T, Wood J A case of cerebral aneurysm rupture and subarachnoid hemorrhage associated with air travel. Open Access Emergency Medicine. 2014;6:23-26.

8. Kouliev T, Richardson A, Glushak C Intracranial hemorrhage during aeromedical transport and correlation with high altitude adaptions in the brain. Open Access Emergency Medicine. 2012;4:93-95.

9. Goldberg CR, Hirschfeld A Hemorrhage within brain tumors in association with long air travel. Acta Neurochir. 2002;144(3):289-293.

10. Zrinzo LU, Crocker M, Zrinzo LV, et al. Commercial flights and patients with intracranial mass lesions: a caveat. Report of two cases. $J$ Neurosurg. 2002;105(4) 627-630.

11. Wilson MH, Edsell M, Davagnanam I, et al. Cerebral artery dilatation maintains cerebral oxygenation at extreme altitude and in acute hypoxiaan ultrasound and MRI study. J Cereb Blood Flow \& Metabolism. 2011;31(10):2019-2029.

12. Jackson M Unruptured intracranial aneurysms in aviation. Aviat Space Environ Med. 2008;79(1):62-64

13. Sirven JL, Claypool DW, Sahs KL, et al. Is there a neurologist on this flight? Neurology. 2002;58(12):1739-1744.

14. Silbergleit R, Burney RE, Draper J, et al. Outcome of patients after air medical transport for management of nontraumatic acute intracranial bleeding. Prehosp Disaster Med. 1994;9(4):252-256. 